\title{
Pulsed Power Modulators for Surface Treatment by Plasma Immersion Ion Implantation
}

\author{
J. O. Rossi, M. Ueda, and J. J. Barroso \\ Laboratório Associado de Plasma, Instituto Nacional de Pesquisas Espaciais \\ Av. dos Astronautas 1758, 12227-010, São José dos Campos, SP, Brazil
}

Received on 15 December, 2003; revised version received on 19 April, 2004

\begin{abstract}
The treatment of surfaces by plasma immersion ion implantation requires pulsed power modulators to provide negative high voltage pulses. To achieve this requirement, we have developed three basic circuit configurations in our laboratory: pulse forming network (PFN), hard-tube pulser (HT) and Blumlein line. In this paper we discuss these three types of circuit topologies.As experimental results, first we present the voltage/current characteristic waveforms of PFN and HT pulsers in the PIII treatment of different materials (aluminum, silicon and stainless steel) as well we describe the surface characterization of the materials thereof treated.And finally,we show recent high voltage tests of a high voltage Blumlein pulser $(150 \mathrm{kV} / 300 \mathrm{~A} / 1 \mu \mathrm{s})$ to be used in surface treatments of polymers and aluminum alloys.
\end{abstract}

\section{Introduction}

PIII process is a technique that was developed during the late 80 s for surface treatment of metallic and plastic materials, which consists of applying negative high voltage pulses to a target immersed in plasma. This technique is a nonline-of-sight method and allows three-dimensional surface treatment of manufactured work pieces with complex shapes. It represents a significant improvement over conventional ion implantation method that requires target manipulation and much more complex systems in such cases. With this technique it is possible to make relevant modifications to the surface properties (i.e. hardness, friction, corrosion resistance, etc) of a wide range of materials such as metals, semiconductors, polymers and ceramics [1], [2]. The PIII process can be applied to semiconductor fabrication, material synthesis, polymers, anti-corrosion coatings, machine tools, metallurgy, etc.

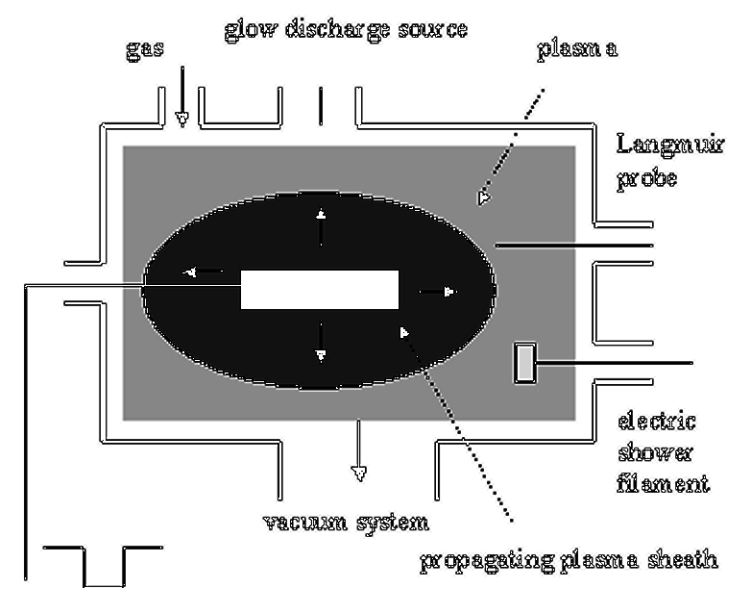

Figure 1. The PIII experimental set-up.
Figure 1 shows the experimental set-up used for the PIII implantation process in our laboratory. The gas containing the ions to be implanted is injected into a vacuum chamber. The most commonly used gas species is $\mathrm{N}$, but it can also be $\mathrm{O}, \mathrm{Ar}, \mathrm{H}$, etc. Here a glow discharge is used to produce the plasma and then a high voltage pulse is applied to the target immersed in the plasma. In fact, the plasma can be generated in two ways: the first one uses DC power supply to apply a constant negative voltage of about $600 \mathrm{~V}$ between a cathode and the chamber wall, while the second employs a microwave source at $2.45 \mathrm{GHz}$ to ionize the gas. In the first method, electrons extracted from the cathode colliding with the gas molecules produce low-density plasma on the order of $10^{10} \mathrm{~cm}^{-3}$. In the second method, transferring the energy from the electromagnetic waves to the electrons ionizes the gas producing high-density plasma on the order of $10^{11} \mathrm{~cm}^{-3}$. A Langmuir probe was used to measure the temperature and the density of the electrons. As the high negative voltage pulse is applied to the sample, a positively charged sheath (non-neutral) region between the plasma bulk and the sample is progressively increased. During the presence of the negative voltage pulse the electrons are repelled from the target on a time scale that is much shorter than the characteristic time of the ion motion. This leaves behind an ion plasma sheath that expands towards the walls of the vacuum chamber. The remaining electric field accelerates the plasma ions towards the target. As a result, the ions are implanted into the target surface from all directions forming a thin material layer. To increase the retained dose (RD) and, consequently, the layer thickness it is necessary to give more energy to the plasma ion by applying a higher voltage. An undesirable RD saturation in glow discharge PIII-based is caused by the removal of the implanted layer (etching effect) due to the high plasma potential (around 350V). One way to reduce this effect is lowering the plasma potential to $70 \mathrm{~V}$ by using a $50 \mathrm{~W}$ electron shower filament as shown 
in Fig. 1. Also this electron shower is used to initiate and maintain the DC glow discharge. Another method consists of increasing substantially the repetition frequency and duration of the high voltage pulse to overcome the etching rate. Despite these disadvantages, the DC glow plasma discharge was used more often because of its easier production compared to the microwave plasma.

\section{Pulsed power modulator circuit configurations}

PIII systems need typically applied voltages in the range of $10 \mathrm{kV}-100 \mathrm{kV}$ with pulse duration between $1 \mu \mathrm{s}-100 \mu \mathrm{s}$ at a repetition frequency of the order of $10 \mathrm{~Hz}-1 \mathrm{kHz}$. To meet these requirements three basic configuration circuits for voltage modulation were tested: pulse forming network (PFN), hard-tube (HT) and Blumlein line. We start this discussion by introducing the HT configuration that has the most widespread use in PIII processes at present [3], [4], [5]. The reason for that is due to three main advantages of this circuit: 1) no need for matching the generator output to the load, 2) adjustable pulse length and 3) high frequency repetition. In this configuration an interruptible on-off switch (tetrode tube) is used to modulate the output voltage by discharging partially a high capacitance $\mathrm{C}$ into the load $\mathrm{R}_{p}$ as shown in Fig. 2(a). The interval time between the closure and opening of the switch defines the pulse duration. The maximum output voltage in the scheme of Fig. 2(a) is generally limited to up to $100 \mathrm{kV}$ because of the maximum blocking voltage of available tetrode tubes. For higher voltages it is necessary to use a pulse transformer to elevate the load voltage at the cost of some increase in the pulse rise-time owing to the transformer leakage inductance. On the other hand pulse transformers allow working with tube blocking voltages of $10 \mathrm{kV}$ to generate high voltage pulses in excess of $100 \mathrm{kV}$ as suggested in [6]. Because of the relatively low current capability of the tetrodes and high cost, the HT pulser is not desirable for high current applications above $10 \mathrm{~A}$ using only one tube. On-off switching devices or tetrode tubes with current capabilities exceeding considerably this value have prohibitive cost and their restricted availability in the market reduces substantially the chance of being used in PIII commercial pulsed modulators. Parallel switching for high current is possible using the tube-MOSFET cascade type connection in which the switching is controlled via a MOSFET transistor in series with the cathodes of several tubes connected in parallel [3], [5]. However, due to practical reasons in connecting many tubes and their cost the pulser current using this configuration cannot exceed several tens of amperes. When compared to other configurations, high costs and the possibility of switch failure are the principal disadvantages of the HT pulser. Since short-circuit currents are difficult to interrupt, a protection circuit or a resistor in series with the load is necessary to protect the tube against a load fault.
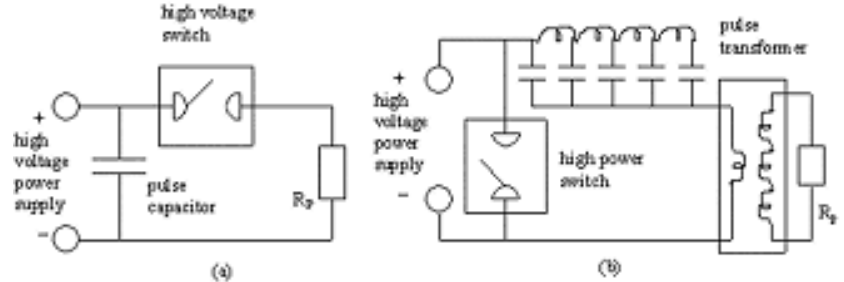

Figure 2. Schematic diagram of two types of conventional pulsers: (a) HT and (b) PFN.

In PFN configuration as shown in Fig. 2(b) a network consisting of discrete inductors $\mathrm{L}$ and capacitors $\mathrm{C}$ is totally discharged into the load [7], [8], [9]. The major advantage of this circuit is that the voltage across the switch decreases to zero at the end of the pulse, which permits the use of a simple non-interruptible gas switch such as a thyratron tube. In this case the line length (number of LC cells in the PFN) determines the pulse duration and the output pulse shape. Although a lumped-parameter network cannot produce an ideal rectangular pulse shape, the overshoot and oscillations in the pulse are reduced to any desired degree by employing a sufficient number of LC sections. The PFN system is adequate for applications with current above 100A since thyratron tube capabilities can reach values on the order of $3 \mathrm{kA}$. Moreover, thyratron tubes are less expensive than tetrode tubes. Because they are also more robust, fault load poses no serious problem in the PFN pulser, which eliminates the need for protection systems. Despite these advantages, there are several drawbacks compared to HT pulsers. First, only increasing the number of LC cells can lengthen the pulse duration. Second, to be efficient PFN circuits must operate with their output matched to the load. To meet this requirement and to increase the output voltage a pulse transformer is usually required. Unfortunately the pulse transformer increases the pulse rise time because of its leakage inductance, being of difficult construction due to both the high voltage isolation needed between primary and secondary coils and the magnetic saturation of high frequency core materials (ferrites) used for pulsed applications.

In several PIII applications it is desirable to operate with voltages in excess of $100 \mathrm{kV}$ depending on target area, plasma density and average output power. As the implanted current depends on $\mathrm{V}^{3 / 2}$ according the Child Law current, by increasing the applied voltage we can obtain higher retained dose of the ions. Moreover, since ions have more energy due to the higher voltage applied, they penetrate deeper into the target. For example, consider the aluminum species, which is an important material used for aerospace industry. Implanting nitrogen $\left(\mathrm{N}_{2}\right)$ ions into $\mathrm{Al}$ pieces enhances tribological properties of their surfaces. However, low energy of the ions has been inadequate for $\mathrm{Al}$ surface treatment because the thick oxide layer formed prior to and during the ion irradiation hinders a proper penetration of nitrogen into the aluminum substrate. Other positive prospect with implantation of high-energy ions is the substantial penetration depth of different ion species (as $\mathrm{Al}, \mathrm{Si}, \mathrm{N}$, etc) into the substrates of polymer-based materials compared to the lowenergy case. In these applications PFN pulsers are adequate because voltages and currents measured over $100 \mathrm{kV} / 100 \mathrm{~A}$ 
can be obtained in these devices. However in PFN systems the main problems are associated with construction of the pulse transformer (mentioned before) and with the capacitor lifetime that is reduced by the occurrence of voltage reversal due to load mismatches. During the PIII process startup, it is very common arc-downs inside the chamber generating voltage reversals of $50 \%$. Also the plasma dynamic impedance varies by a typical factor of 10 during the implantation process, which produces substantial voltage reflections that decrease the capacitor lifetime [10]. In view of that, we have been working on a high voltage pulser based on Blumlein topology [11], [12] shown in Fig. 3, which uses a gas switch (such as a thyratron tube) and eliminates the need of conventional capacitors and pulse transformers. This circuit design is a version of the common voltage inversion generator called stacked Blumlein line generator proposed by Fitch and Howell [13], where pulse forming lines (made of striplines or coaxial cables) are charged in parallel and discharged in series to produce the high voltage pulse across the load. Fig. 3 illustrates the principle of operation of a coaxial device where 10 coaxial lines are charged simultaneously from a negative charging voltage $-\mathrm{V}$. It can be summarized as follows: in the initial state the lines are charged, but the net output voltage is zero because of the initial voltage vector opposition as indicated in Fig. 3. As soon as the switch is closed, pulse voltages propagate down the active lines on the left. After one propagation delay time they arrive at the output where reflections occur converting the initial vector opposition to a series addition, which persists for twice the line propagation time. This leads to an output voltage of $-\mathrm{nV}$ for an open end or else $-n V / 2$ for the case of a load $n Z_{0}$ matched to the generator output, where $\mathrm{n}$ is the number of lines and $\mathrm{Z}_{0}$ is the line characteristic impedance. Regarding the pulser configuration circuit, there are two important impedances in a cable, the inner cable characteristic impedance $\mathrm{Z}_{0}$ and the impedance $\mathrm{Z}_{2}$ of the outer part of the shield as shown in Fig. 3. If $Z_{2}<<Z_{0}$ a fraction of the output current can reach ground via cable shielding without going through the load. However, if the outer impedance $\mathrm{Z}_{2}$ can be made large, for example by winding the cable on a coil former so that the inductance of the outer part of the jacket is high, then it reduces the drained current and, consequently, the overall power loss of the device. This is an important aspect design to be implemented during the pulser construction because of two reasons: 1) it improves the voltage gain of the device and 2) increases the pulser compactness as long lengths of lines are not extended in a straight line. Compared to conventional PFN and HT pulsers in high voltage applications, the main advantages of Blumlein systems are their simpler construction and lower cost. For instance, replacement of cables is cheaper than capacitors or transformers in case of voltage breakdown in the dielectric caused by voltage reflections. Nevertheless in Blumlein circuits (such as in PFNs) the pulse length is fixed (depends on the line length) and load matching is necessary for a rectangular pulse shape. Besides that, for operation in the microsecond range long lengths of cables are required.

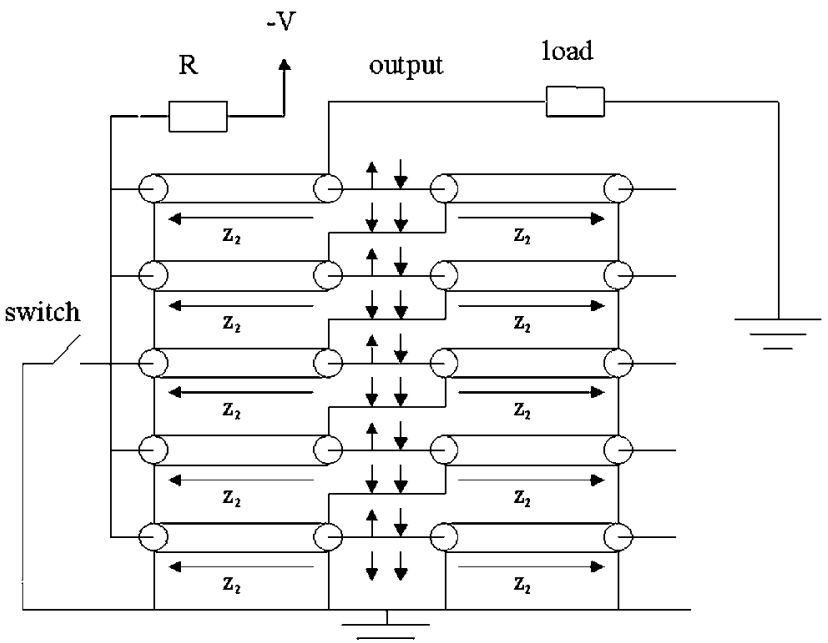

Figure 3. A five-stage Blumlein coaxial pulser made of ten lines.

\section{Experimental results}

At our laboratory we started the PIII experiment by using a PFN pulser to feed a PIII system based on high-density plasmas produced by a $2.45 \mathrm{GHz}$ microwave source [8], [9]. Then we continued the tests employing a HT pulser to drive low-density plasmas generated by a DC glow discharge [4], [5]. In the next subsections we will present a brief description of these pulsers giving some results obtained with PIII treatments in aluminum, stainless steel and silicon samples. At last, we describe the Blumlein device under development for high voltage applications on the order of $100 \mathrm{kV}$ to be used in treatment of polymer and aluminum materials, presenting a high voltage pulse test into a matched load.

\subsection{Results with PFN pulser}

The PFN was built by using ten sections (see Fig. 2(b)) with $\mathrm{L}=300 \mathrm{mH}$ and $\mathrm{C}=2.5 \mathrm{nF}$, which gives an output impedance $\mathrm{Z}_{0}=(\mathrm{L} / \mathrm{C})^{1 / 2}=350 \Omega$ and pulse duration $\mathrm{T}=2 \mathrm{~N}(\mathrm{LC})^{1 / 2}=20$ $\mu \mathrm{s}$, where $\mathrm{N}$ is the number of LC cells. The pulser charger was a current constant switching power supply of $50 \mathrm{kV} / 10 \mathrm{~mA}$. Because of the low current capability of the charger (10mA only) the pulse repetition frequency was limited to up to only $20 \mathrm{~Hz}$. The charging operation finishes when the voltage on the network is close to the selected charger voltage. The PFN was discharged into the load by means of a fast switch thyratron tube $5 \mathrm{C} 22(16 \mathrm{kV} / 300 \mathrm{~A})$. To avoid problems in the high voltage isolation and ferrite saturation, an air-core pulse transformer with turn ratio $n=2$ was used to match the PFN characteristic impedance to the plasma resistance. As the primary impedance is reflected to the secondary side of the transformer by factor of $n^{2}$, a plasma resistance on the order of $4 \mathrm{Z}_{0}=1400 \Omega$ was estimated during the implantation process as shown in the output voltage-current characteristic of the PIII reactor driven by the pulser in Fig. 4. In this pulser system, the output voltage is approximately equal to the PFN charging voltage since the transformer ratio is 2 and for a matched load the voltage 
across the primary side of the transformer is half the PFN voltage. The PIII reactor consisted of a stainless steel vacuum chamber with a cylindrical volume $(0.28 \mathrm{~m}$ diameter and $0.38 \mathrm{~m}$ length). The microwave radiation produced by a commercial magnetron source is transmitted through a glass window. At an operating pressure in the range of $10^{-4}$ mbar the input radiation breaks down the gas, forming dense nitrogen plasma. The main parameters of the plasma obtained inside the chamber were measured by means of a Langmuir probe, which indicated an electron temperatures on the order of $1-10 \mathrm{eV}$ and plasma densities of $10^{11} \mathrm{~cm}^{-3}$, at operation pressures in the range of $1-7 \times 10^{-3}$ mbar. The extraction of $\mathrm{N}^{+}$ions from the plasma was accomplished by applying the PFN negative high voltage pulses (in the range of $10-20 \mathrm{kV}$ and $5-20 \mathrm{~Hz}$ ) to the sample. Fig. 5 shows typical voltage and current waveforms obtained during the operation of the PIII reactor. In this case we notice pulse voltage distortion caused by the parasitic effects of the transformer leakage inductance and plasma sheath capacitance. The combination of leakage inductance with the plasma sheath capacitance increases the pulse rise time while the discharge of the sheath capacitance into the plasma bulk lengthens the pulse fall time, deforming the rectangular pulse shape.

For the PFN tests, A15052 samples have been bombarded with nitrogen ions in the range of $10-1 \mathrm{keV}$ [14]. For example, as a preliminary result micro-hardness tests with Knoop indentation on Al5052 at retained doses on the order of $10^{15} \mathrm{~cm}^{-2}$ showed improvement in surface hardness $(0.64 \mathrm{GPa})$ compared with non-implanted surfaces $(0.57 \mathrm{GPa})$. Also $\mathrm{x}$-ray diffraction for the same material and estimated dose showed increased stress in its structure but no formation of AlN phases which requires doses of about $10^{17} \mathrm{~cm}^{-2}$.

\subsection{Results with Hard-Tube pulser}

For the PIII processing we have used the basic HT circuit configuration as shown in Fig. 6, where the cathode of the tube operates floating at a high negative potential. A DC high voltage power supply charges the capacitor $C$ while the resistor $\mathrm{R}_{1}$ limits the transient charging current. Also a TTL pulse generator located on ground at the panel rack controls the pulse length. The tube remains switched on as long as the TTL pulse is present and whenever an external triggering occurs, the potential on the tetrode control grid is increased from $-300 \mathrm{~V}$ (cut off condition) to a few volts above the tetrode cathode potential leading the tube into saturation. As the tube and its driver amplifier are located on the high voltage floating deck, an optical fiber transmits the TTL signal via optical interface units. A second resistor $R_{2}$ of $6 \mathrm{k} \Omega$ in series with the tetrode and the plasma load protects the system against the risk of short-circuits and eliminates protection circuit complexity but at the expense of increasing pulse rise time. Since the current capability of the tetrode is of the order of $10 \mathrm{~A}$ in the case of a load fault, the limit of the charging voltage is up to a maximum of $60 \mathrm{kV}$.

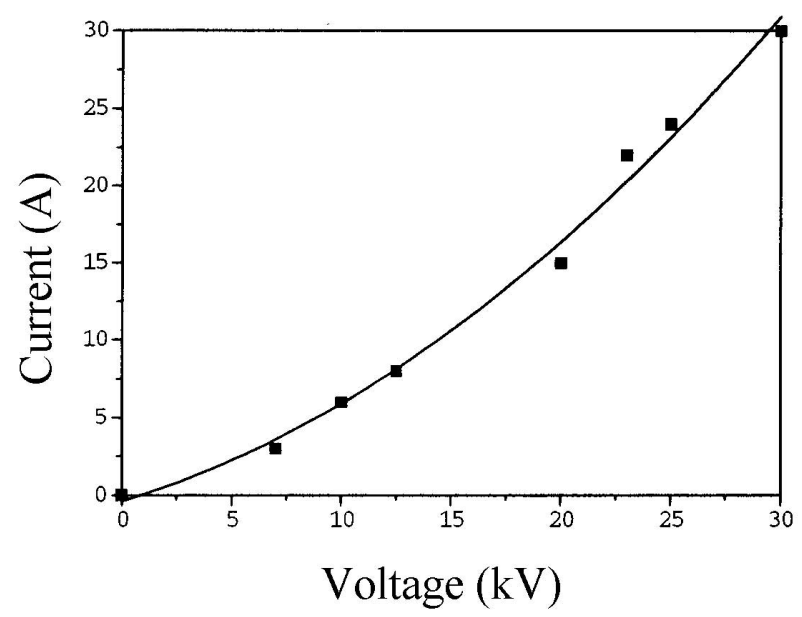

Figure 4. Output voltage-current characteristic curve of the PIII reactor driven by the pulser.

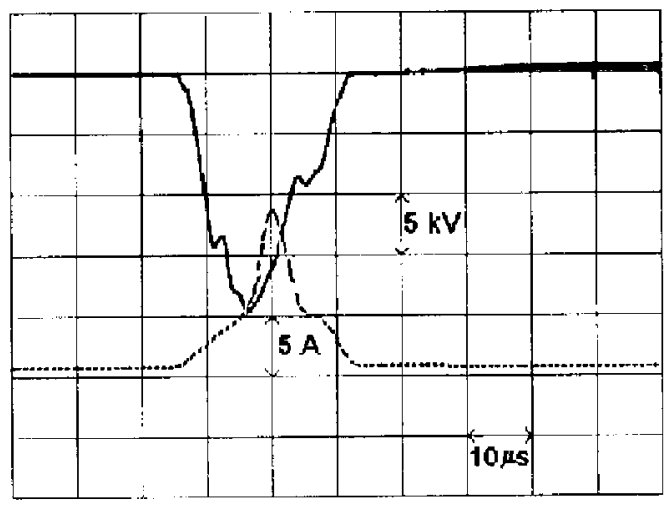

Figure 5. Pulse voltage (top) and current (bottom) waveforms obtained during the operation of the PIII reactor.

As in the case of the PFN pulser, HT pulse generator was connected to the same plasma vacuum chamber. Several samples of different materials such as Si and SS304 were immersed in the nitrogen plasma [15]. Using a DC power supply of $600 \mathrm{~V} / 1 \mathrm{~A}$, a glow discharge between a small electrode located inside the chamber and the ground (the chamber walls) produced plasma of lower density in this case. A Langmuir probe measured the plasma density of $\mathrm{n}_{e}=2.5 \times 10^{10} \mathrm{~cm}^{-3}$ and electron temperature of $\mathrm{T}_{e}=5.0 \mathrm{eV}$ while an ion gauge meter indicated a plasma pressure of $0.8 \times 10^{-3}$ mbar with pulse repetition rates of approximately $700 \mathrm{~Hz}$ attained during the PIII treatment. Fig. 7 shows the typical voltage (top) applied to a SS304 target during the implantation process and the corresponding pulse current (bottom) for the HT pulser. From the voltage waveform, we 
observe the pulse generator was adjusted for output pulse amplitude on the order of $12 \mathrm{kV}$ and duration of $50 \mu \mathrm{s}$. Also note the long fall and rise times of the output voltage hinders the pulse shape leading to an approximate triangular form. As mentioned before, this is caused by two parameters: 1) the high value of the protection resistor $(6 \mathrm{k} \Omega)$ and 2$)$ the capacitance of the output coaxial cable that connects the generator to the PIII facility $(3.0 \mathrm{nF})$, which produces a high $\mathrm{RC}$ constant during the fall and rise of the pulse. Moreover, as shown in voltage/current curves of Fig. 7 the pulse fall is exponential and this can be explained by taking into account the sheath capacitance discharge into the plasma bulk resistance after the opening of the switch.



Figure 6. Schematic diagram of the $60 \mathrm{kV} / 10 \mathrm{~A}$ hard-tube pulser.

Using this type of pulser we have obtained successful treatment of SS304 samples [16] with deep penetration of nitrogen of about 200nm and peak concentrations of about $35 \%$ atomic concentration, using Auger electron emission spectrometry (AES). In this case for an exposition time of $15 \mathrm{~min}$, RD larger than $1 \times 10^{18} \mathrm{~cm}^{-2}$ was achieved. Using a higher exposure time of $60 \mathrm{~min}$, the obtained AES profile indicated a penetration of more than $1 \mathrm{~mm}$ and higher RD of about $4 \times 10^{18} \mathrm{~cm}^{-2}$ with the formation of nitrides or nitrogen saturated expanded austenite phase evidenced by $\mathrm{x}-$ ray diffraction peaks near to the usual $\gamma$-Fe patterns. Such high doses are sufficient to produce radical changes in tribological properties of the surfaces of these SS304 targets. In fact, preliminary tests in a nanohardness indenter device revealed a hardness factor of the treated sample 2.5 higher compared with that of a non-implanted one. The second important result obtained is related to nitrogen implantation into silicon wafers. The atomic concentration profiles produced by AES measurements indicated that RD increases strongly from $3 \mathrm{~min}$ to $15 \mathrm{~min}$ (reaching $3 \times 10^{17} \mathrm{~cm}^{-2}$ at maximum) and drops afterwards. Also these profile results showed strong etching of the substrate surface for an exposition time $\mathrm{t}>15 \mathrm{~min}$. The etching depths were confirmed by the Si surface profile measurements, being on the order of $80 \mathrm{~nm}$ for $60 \mathrm{~min}$ case. In the case with highest RD $\left(3 \times 10^{17} \mathrm{~cm}^{-2}\right)$ compounds such as $\mathrm{SiO}_{2}$ and $\mathrm{Si}_{3} \mathrm{~N}_{4}$ were identified and their profiles determined by AES technique.
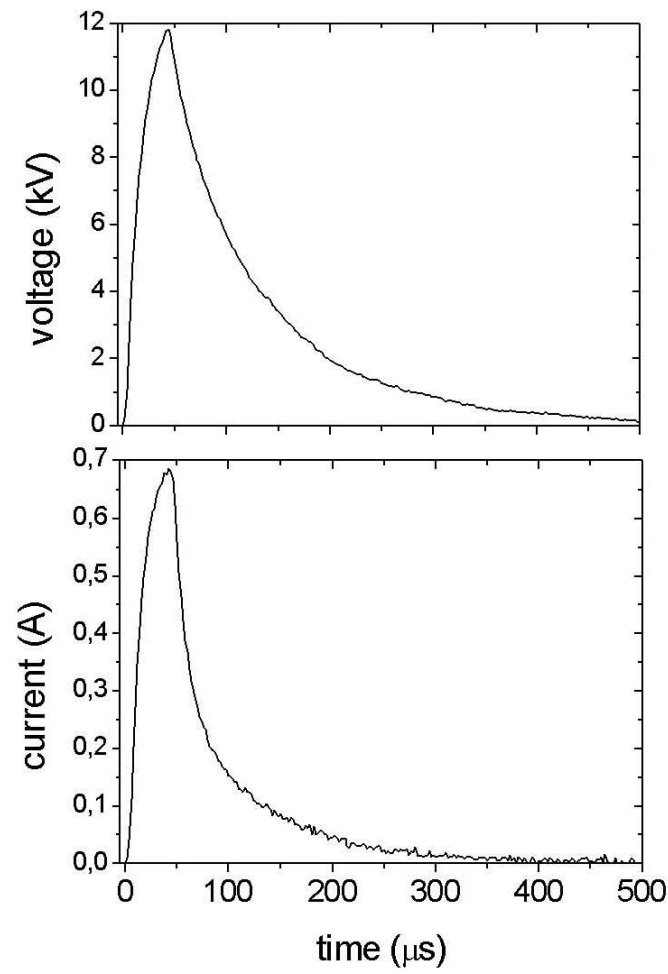

Figure 7. Pulse voltage (top) and current (bottom) waveforms during the PIII process for the HT pulser.

\subsection{Tests with Blumlein pulser}

The requirements of our pulser design are: $150 \mathrm{kV} / 300 \mathrm{~A}$ with pulse duration of $1 \mu \mathrm{s}$ [17], [18], [19]. Thus the pulser load of $500 \Omega$ requires a design with 10 pieces of a $50 \Omega$ coaxial cable (or 5-stages). To ensure a high breakdown voltage, we chose the coaxial cable URM67/50 $\Omega$ with the maximum rating of $40 \mathrm{kV}$. Considering a pulser with 10 lines (i.e., with an ideal gain of $n=5$ ) and its output nominal voltage of $150 \mathrm{kV}$ gives a maximum charging voltage of $30 \mathrm{kV}$, below the cable breakdown voltage. The length of each transmission line is $100 \mathrm{~m}$ as the selected cable URM67 has a double transit time of $10 \mathrm{~ns} / \mathrm{m}$, which implies in the pulse duration of $1 \mu \mathrm{s}$. To increase the device gain efficiency, the coaxial lines were wound around cylindrical tubes, made from PVC and supported by an aluminum structure. In practice, we have estimated the shield cable impedance $\mathrm{Z}_{2}$ by measuring the inductance $\mathrm{L}$ and capacitance $\mathrm{C}$ (with respect to ground) of one coaxial winding in the pulser structure. By using an LC bridge meter we obtained an inductance of about $1.0 \mathrm{mH}$ for each coaxial coil. In relation to $\mathrm{C}$, we verified that the meter provided approximately the same capacitance (on the order of $250 \mathrm{pF}$ ) for each coaxial coil with respect to ground. Calculating through the well-known formula for the line characteristic impedance gives $Z_{2}=2 \mathrm{k} \Omega$. 
To charge the coaxial cables we use an especial switchedmode power supply for constant current charging with maximum output voltage capability of $35 \mathrm{kV}$ and charge rate of $8 \mathrm{~kJ} / \mathrm{s}$, which is able to provide a maximum repetition frequency in the range of $100 \mathrm{~Hz}$ according to the pulser specifications. For the switching system a thyratron tube with voltage/current ratings of $35 \mathrm{kV} / 5 \mathrm{kA}$ was used. This switch incorporates a glass envelope single gap tube that conducts in the reverse direction (hollow anode version), which is an important characteristic for the pulser design as the impedance mismatch into the system caused by the shield cable impedance produces negative voltages reflected at the generator input. Moreover, as the plasma resistance varies linearly during the pulse application (predicted by the plasma modeling) the performance generator deteriorates because of the impedance mismatch. The main consequences are the gain drop and long rise time of the applied voltage pulse, the implantation current decrease (instead of flat top pulse) and the presence of voltage and current reflections. Despite this, the pulse generator operation for plasma processing is possible since the most part of the energy is spent into the load plasma load resistance during the implantation process since two main process parameters such as plasma density and target area be adjusted [20].

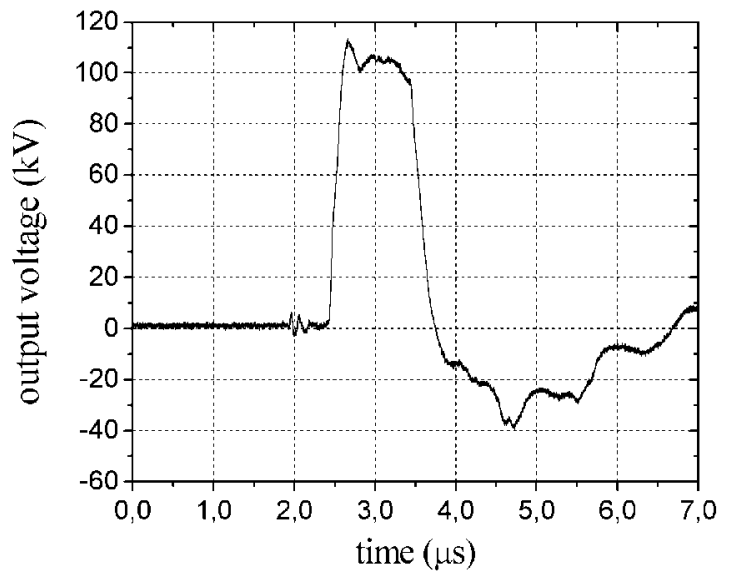

Figure 8. Pulse output voltage produced by the Blumlein Pulser for a charge voltage of $25 \mathrm{kV}$.

For the initial tests of the pulser we used a load resistance of about $500 \Omega$. Fig. 8 shows the output voltage pulse obtained with positive polarity in single shot for an initial charging voltage on the order of $25 \mathrm{kV}$, which gives a pulser gain efficiency in the range of $80 \%$. From this figure, we can observe that the pulse rise time is less than $200 \mathrm{~ns}$ with a pulse length around 800ns. Also note that the negative voltage swing in the tail of pulse is caused by the impedance unbalance due to the cable shield impedance $\mathrm{Z}_{2}$ in the pulse generator structure. Besides that, we observed that during these high voltage tests as soon as the voltage across the matched load reaches up to $50 \mathrm{kV}$ the losses increase and the gain efficiency drops from $90 \%$ to $80 \%$ because of the corona effects and induced currents into the metallic structure of the generator. However, to reach the specified value of operation $(150 \mathrm{kV} / 100 \mathrm{~Hz})$ the losses caused by flashover discharges and induced currents should be minimized. If we will be able to suppress or to decrease these additional losses the pulser efficiency drop is minimized, being at least on the order $10 \%$ due to the inherent presence of $Z_{2}$. Finally, Fig. 9 shows a general view of the Blumlein generator (to the left) and the vacuum chamber of the PIII facility (to the right) to be integrated with the pulser.

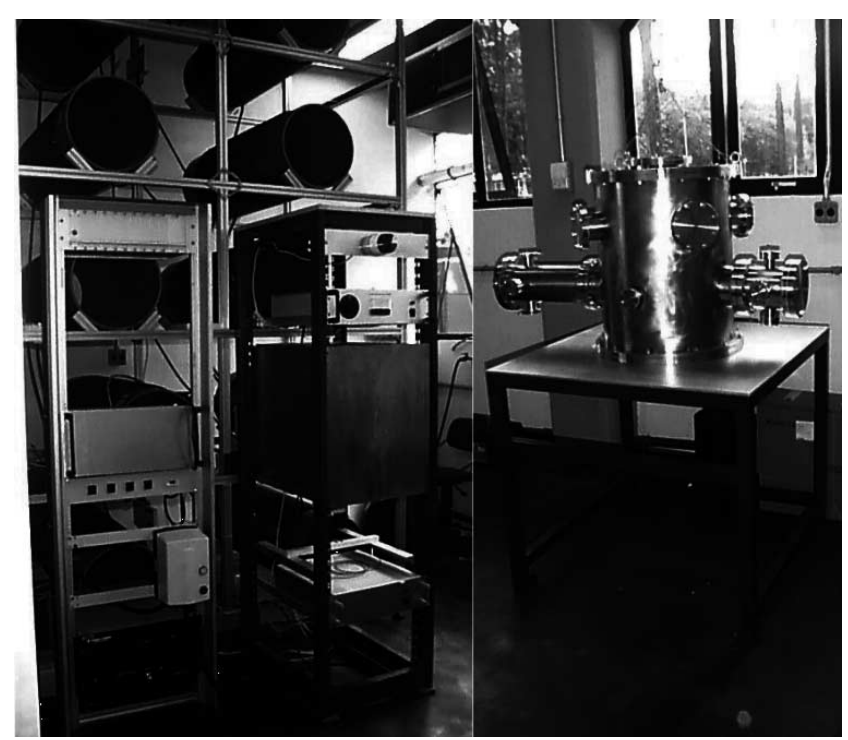

Figure 9. General view of the Blumlein pulser (left) and the plasma chamber (right).

\section{Conclusions}

We have presented three basic types of circuits for pulse power modulators commonly employed in surface treatments by plasma immersion ion implantation (PIII). Two of them, pulse forming network (PFN) and hard-tube (HT) configurations have been used in our laboratory with great success. At present, because of the excellent results given by a HT pulser in the treatment of SS304 samples and Si wafers we have discontinued the PFN, focusing our research on the use a commercial HT pulse generator (RUP-4) with higher current capability and frequency $(30 \mathrm{kV} / 30 \mathrm{~A} / 1 \mathrm{kHz})[5]$ to be applied to the treatment of industrial work-pieces as fast steel drill bits, knife blades, tools for wood cutting, prosthesis made of Ti alloy, etc [21]. At the same time, we are developing a new configuration circuit based on Blumlein generators to be used in PIII treatment of polymer and Al surfaces with nitrogen ions of high energy (of about 100keV). First, we have tested the device using a load resistance obtaining an output pulse voltage of about $100 \mathrm{kV}$ with a pulse length on the order of $800 \mathrm{~ns}$, pulse rise time less than $200 \mathrm{~ns}$ and gain efficiency of $80 \%$. As a result, we expect to reach the specified voltage of $150 \mathrm{kV}$ by minimizing the pulser losses and, consequently, increasing the device gain efficiency up to $90 \%$. Moreover, we intend to complete the assembling of the chamber by installing the vaccum system and the voltage/current feed-through in order to start the implantation of high-energy $\mathrm{N}_{2}$ ions into polymers and aluminum alloys. 

PESP.

The authors acknowledge financial support from FA-

\section{References}

[1] J. L. Shohet, IEEE Trans. Pla. Sci. 19(5), 725 (1991).

[2] J. R. Conrad and J. L. Radke. J. Appl. Phys. 62(11), 4591 (1997).

[3] J. Brutscher, Rev. Sci. Instrum. 67(7), 2621 (1996).

[4] J. O. Rossi, M. Ueda, and J. J. Barroso, and V. A. Spassov, IEEE Trans. Pla. Sci. 28(5), 1392 (2000).

[5] J. O. Rossi, M. Ueda, and J. J. Barroso, Surf. Coat. Technol. 136, 43 (2001).

[6] G. Böhm and R. Günzel, J. Vac. Sci. Technol. B 12(2), 821 (1994).

[7] G. N. Glasoe and J. V. Lebacqz, Pulse Generators (McGrawHill, New York, 1948).

[8] V. A. Spassov, J. Barroso, M. Ueda, and L. Guerguiev, Astrophys. and Space Sci. 256, 533 (1998).

[9] V. A. Spassov, M. Ueda, and J. Barroso, Surf. Coat. Technol. 112, 29 (1999).

[10] W. A. Reass, J. Vac. Sci. Technol. B12(2), 854 (1994).

[11] J. O. Rossi, M. Ueda, and J. J. Barroso, AIP Conference Proceedings, vol. 563, 96-101, edited by $\mathrm{H}$. Chuaqui and M. Favre (AIP, New York, 2001).
[12] J. O. Rossi, M. Ueda, and J. J. Barroso, IEEE Trans. Pla. Sci. 30(5), 1622 (2002).

[13] R. A. Fitch and V. T. S. Howell, Proc. IEE 111(4), 849 (1964).

[14] M. Ueda, C. Stellati, V. A. Spassov, J. J. Barroso, and M. C. A. Nono, Surf. Coat. Technol. 112, 310 (1999).

[15] M. Ueda, G. F. Gomes, L. A. Berni, J. O. Rossi, J. J. Barroso, A. F. Beloto, E. Abramof, and H. Reuter, Nucl. Intrum. Meth. Phys. Res. B 161-163, 1064 (2000).

[16] M. Ueda, L. A. Berni, J. O. Rossi, J. J. Barroso, G. F. Gomes, A. F. Beloto, and E. Abramof, Surf. Coat. Technol. 136, 28 (2001).

[17] J. O. Rossi, M. Ueda, and J. J. Barroso, WSEAS Trans. Syst. 1(2), 24 (2003).

[18] J. O. Rossi, M. Ueda, and J. J. Barroso, in 14th Int. Pulsed Power Conf. 15-18 June 2003, TX, USA, 533.

[19] J. O. Rossi, M. Ueda, and J. J. Barroso, in Brazilian Power Electronics Conf., 21-24 Sept. 2003, Fortaleza, CE, 521-523.

[20] J. O. Rossi, M. Ueda, and J. J. Barroso, in Brazilian Power Electronics Conf. 11-14 Nov. 2001, Florianópolis, SC, vol.2, 569-553.

[21] M. Ueda, L. A. Berni, R. M. Castro, A. F. Beloto, E. Abramof, J. O. Rossi, J. J. Barroso, and C. M. Lepienski, Surf. Coat. Technol. 156, 71 (2002) 